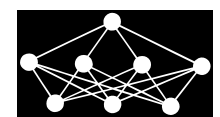

\title{
NETWORK MODELS FOR CHANGING DEGREE DISTRIBUTIONS OF FUNCTIONAL BRAIN NETWORKS
}

\author{
M. Markošová, B. Rudolfł P. Náther, $\check{L}$. Beňušková*
}

\begin{abstract}
The purpose of this study was to investigate degree distributions of functional brain networks. Particular functional brain networks were constructed from the fMRI measurements of three groups of participants namely, young healthy participants, elderly healthy participants and elderly participants with Alzheimer disease. Functional brain networks were constructed for three different correlation thresholds of voxel activity correlated over time. We have noticed that the character of degree distribution changes when the value of correlation threshold decreases. In order to explain the degree distribution changes with the changes of value of correlation threshold, we created two different, yet related network models. The crucial factor both models contain is an increasing noise as the voxel activity correlation threshold is lowered, which in our models corresponds to an increase of the number of random correlations between the voxels - nodes of the functional network. The models account for how initially scale-free character of the degree distribution changes as the correlation threshold is lowered based on the processes of network growth and edge addition. The two models differ in the manner of preferential and random edge addition while the second model is a refinement of the first one. On average, the second model leads to a better quantitative match with the data. To our knowledge, such functional brain network models, which take into account the correlation threshold as an independent variable have not been introduced before.
\end{abstract}

Key words: network model, fMRI, functional brain networks, correlation threshold, noise

Received: May 6, 2019

DOI: $10.14311 / N N W .2020 .30 .021$

Revised and accepted: October 30, 2020

*Mária Markošová; Peter Náther; Ľubica Beňušková - Corresponding author; Department of Applied Informatics, FMFI UK, Mlynska dolina, 84248 Bratislava, Slovakia, E-mail: lubica@ii. fmph.uniba.sk

${ }^{\dagger}$ Boris Rudolf; Institute of Mathematics and Informatics, Faculty of Electrical Engineering, Slovak University of Technology, Bratislava, Slovakia 


\section{Introduction}

\subsection{Functional brain networks derived from fMRI treated as graphs}

Functional Magnetic Resonance Imaging (fMRI) is a non-invasive measurement technique, which captures high resolution images of neural activity in the brain with an acquisition time of around 1-3 seconds. The level of neural activity is inferred from the blood-oxygen-level-dependent (BOLD) signal [7]. Recorded fMRI images consist of a series of three dimensional slices representing cross sections of the brain in a serial order. Each slice is comprised of a rectangular grid of discrete $3 \mathrm{D}$ regions known as voxels (volumetric pixels) of about $3 \mathrm{~mm}^{3}$ or less, depending on the scanner. A full 3D spatial image of the brain activity is a result of combining all the slices together. Since fMRI data is comprised of fMRI time-series data of activity of thousands of voxels, fMRI is often used for deriving functional connectivity of the brain based on voxels exhibiting similar activity over time [32]. By modeling this functional connectivity as a network (i.e. functional brain network), we have decided to use techniques from the graph theory to explore their topological characteristics. In the network analysis of the functional brain networks, different anatomical brain regions can become the nodes. However, we have chosen the individual voxels as nodes of the functional brain network. As for the edges, the most commonly used measure of similarity is correlation, which is based upon the concept of covariance. Covariance is a measure of the extent to which two random variables vary together or, in other words, a measure of their dependence: the extent to which knowledge of one variable could be used to predict the other.

Mathematical representation of the network is a graph. Graph $G(V, E)$ consists of $N=|V|$ nodes, connected by $m=|E|$ edges [35], while $V$ and $E$ denote node and edge sets respectively. In this study we will deal with the so-called binary networks, which are networks having undirected unweighted edges. An important notion is the degree of a node, which is the number of edges incident with the node in question. The degree distribution is the probability distribution of the degrees over the whole network. The most elaborated graph theory is that of random graphs [12]. However, due to the specific properties of real-world networks, random graphs are often not a good model for them. As has been shown by Barabási and Albert [3] and others [10,19,28,34], real-world networks, like social networks, Internet, language lexicon, etc., have usually small-world and scale-free character, and other properties like e.g. modularity and others.

We were interested in topological characteristics of the brain functional networks, which are real-world complex networks, especially whether they have a scale-free character. This property is reflected in the degree distribution, which has a power law character. For the normalized number of nodes with the degree $k$, the probability $P(k)$ decreases as a power law with the scaling exponent $\gamma$, i.e.

$$
P(k) \propto k^{-\gamma}
$$

Barabási and Albert [3] showed that the scale-free structure develops due to a specific network dynamics. This dynamical principle, based on a preferential node linking, is common for many real-world networks and can be expressed as "the rich 
get richer" rule. If a new node comes to the network, it links to the node with a high degree with higher probability than to a node with a small degree. Two seminal papers in the complex network analysis by Watts and Strogatz [34] and Barabasi and Albert [3], triggered a wide range of work across many disciplines in search to find evidence of 'small-world' and 'scale-free' networks in both the natural and manmade worlds. Other influential papers introduced complex measures for network analysis $[10,26]$. These papers also helped to popularize the use of networks, or graphs, as the data structure of choice for many forms of data analysis. Networks are also an intuitively appealing structure to analyze functional connectivity of the brain: Anatomical parts of the brain or voxels are represented as nodes in a network, and interaction (e.g. correlation) between them as edges [15,25].

Eguiluz et al. [11] was one of the first who used fMRI to extract functional networks connecting correlated voxels while subjects were performing different tasks like finger tapping or listening to music. By using a correlation measure between any pair of voxels they built a correlation matrix that was thresholded to construct large-scale brain networks with thousands of nodes. They found that these networks were scale-free networks with power law degree distributions, i.e. Eq. (1), with $\gamma \sim 2$. On the other hand, Achard et al. [1] analyzed fMRI time series acquired from healthy subjects in the resting state. They found that these networks do not display a scale-free topology. Instead they found that the best fit for the degree distribution was an exponentially truncated power law, i.e.

$$
P(k) \propto k^{-\gamma} \mathrm{e}\left(-k / k_{c}\right)
$$

with exponent $\gamma=1.8$ and cutoff degree $k_{c}=5$. There is a theoretical work to account for these results, namely by Portillo and Gleiser [27]). In their model, anatomical regions in the brain are represented by microscopic nodes. To account for the latter degree distribution Eq. (2), they start from a small random network, which grows by the addition of new nodes with fixed number of connections. The new nodes are linked at random, but then the connections are adaptively rewired according to coherence between the nodes. They show that the model is able to describe topological characteristics of the human brain networks obtained from fMRI studies described by Eq. (2). However, most of the studies of functional brain networks derived from fMRI data lead to networks, which have a small-world character but not the scale-free topology $[4,32]$. Thus, there is a discrepancy in the overall topological studies of the brain functional networks. Some studies show they are scale-free [11], some show they have a degree distribution described by an exponentially truncated power law [1] and some claim there is no scale-free property at all [4].

We have noted an important fact that in all the above mentioned studies, the authors used experimental data of various origin (data acquisition parameters, participants of different ages, different preprocessing and analysis methods), obtained under different conditions (task-free, different tasks), and using different methods of brain functional networks derivation including different thresholds for thresholding the correlated activity between the network nodes. However in this study, we concentrate on the effect of thresholding the correlated voxel activity upon the scale-free topology of the functional brain networks derived from a coherent fMRI dataset obtained and derived under the same conditions from three different groups of participants. 


\subsection{The goals}

Our aim in this paper is to study and model degree distributions of functional brain networks by means of the methods of the graph theory. We use the functional brain networks derived from the brain fMRI data of healthy young (HY) participants, healthy elderly participants (HE) and elderly participants with Alzheimer disease (AE). The original data were collected by by Buckner et al. [5]. All participants were performing the same visual motor task. In particular our goals are:

- First, to analyze the degree distributions of functional brain networks. The goal was to find out whether there are differences in their characteristics depending on the age / health state groups of participants, i.e. HY, HE and AE, depending on different correlation thresholds of voxel activity.

- Second, to develop unifying network models that explain the changes in the degree distributions at different correlation thresholds for all groups of participants.

- Third, to test by computer simulations, which model accounts better for changing degree distributions at different correlation thresholds.

\section{The Data}

\subsection{The fMRI Dataset}

Buckner et al. [5] acquired the raw structural and functional MRI data from 41 subjects in total (data set no. 2-2000-118W from the fMRI Data Center: http://www.fmridc.org). In their study, there were three groups of participants: healthy young (HY), healthy elderly (HE) and elderly with diagnosed Alzheimer's disease (AD) of mild or very mild severity (AE) [18]. The HY group had 14 subjects (9 females/5 males) with the mean age 21.1 years (SD 2.0). The HE had 15 subjects (9 females/6 males) of the mean age 75.1 years (SD 6.9). The AE group had 12 subjects ( 7 females/5 males) of the mean age 77.1 years (SD 5.3). There was no statistically significant difference in the mean age of the latter two groups. The participants in the $\mathrm{HE}$ and AE groups were clinically assessed for the presence of dementia using the Clinical Dementia Rating (CDR) [16]. All individuals in the HE group had a CDR $=0$. Of the 12 individuals in the AE group, seven had CDR $=0.5$, corresponding to a diagnosis of probable $\mathrm{AD}$; the remaining five had $\mathrm{CDR}=$ 1, corresponding to a diagnosis of mild AD. Each participant underwent four fMRI recording sessions performing a simple visual motor task. This valuable dataset of three groups of participants of different age and brain health condition has later been used in numerous studies, and has generated a wide range of findings (see e.g. $[8,13,24,33])$.

One of disadvantages of the fMRI method lies in a fact, that the fMRI signals are extremely noisy and the signal to noise ratio is rather low. BOLD related changes generally make up only a few $(\sim 5)$ percent of the overall signal [7]. Two main sources of noise are in question: noise caused by the scanner itself and various types of physiological noise (breathing, heart beat, even a very subtle movement 
of the subject in the scanner causes a problem, etc.). That is why, the data are prepossessed before being used in subsequent studies. Preprocessing applied to the fMRI data [5] is described in detail in [23]. It consisted of the steps briefly listed below, together with references for more detailed information:

1. The first step in data preprocessing consisted of slice-timing correction. Since each slice of fMRI data is taken in a different time, the Fourier interpolation technique was used provided by the 3dTshift tool, namely AFNI [9].

2. To correct for scanner drift, a high-pass temporal filter was applied to every volume, with a pass frequency of $1 / 42.88 \approx 0.02 \mathrm{~Hz}$ with the fslmaths utility, provided with FSL [31].

3. There is always a motion, even a slight one involved in the scanning. Thus, the motion correction was applied to each volume using the FSL's mcflirt utility [17].

4. The next step of preprocessing was the so-called intra-session alignment. The fMRI images from every session were aligned to the first image of the first session in order to allow for averaging across sessions and trials. For that the flirt utility provided by FSL is used.

5. During the process of brain segmentation, the non-brain matter, e.g. skull tissue, was removed from the structural MRI image. The bse utility provided with BrainSuite was used for this purpose [29].

6. To extract voxels that belong to the brain tissue, the tissue classification was performed. Each MRI voxel was classified as white matter, grey matter or cerebrospinal fluid, based on field inhomogenities, using BrainSuite's bfc and pvc utilites. In our current work we used functional networks consisting of the voxels of gray and white matter only.

7. Each participant's brain is different. Thus, the next step, the intra-subject registration, deals with this problem. Using the FSL's flirt utility, a mean fMRI image was created from every structural fMRI volume.

8. After creating the mean fMRI image, the spatial normalisation was applied. First, using FSL's flirt, a 12 parameter affine transformation from the structural MRI image to the $2 \mathrm{~mm}^{3}$ MNI152 T1 standard brain template was calculated. This transformation was then used to initialise a non-linear transformation using the fnirt utility also provided with FSL [2].

\section{$2.2 \quad$ Functional brain networks}

The prepossessed fMRI data were used in [23] to create functional brain networks for each participant in all three groups (HY, HE, AE). The same functional brain networks are the basis of novel analysis performed in the present work. Preliminary analysis and results were published in [21,22]. These networks are unweighted and undirected loopless networks. The process of creation of these functional networks is briefly described below. 
Functional brain networks are based on temporal correlation of activity between the voxels [11]. Thus, the derived functional brain network exists only during given time when the temporal correlation of activity is calculated. During this time, the brain may be involved in performing some task. Thus, the derived functional network reflects the functional cooperation of different brain areas. Because the smallest activity unit of the fMRI brain volume is the composite signal of neurons contained in one voxel, voxels are natural and most appropriate choice for the nodes of functional brain network as argued by Hayasaka and Laurienti [14]. If any two voxels functionally cooperate, based on the underlying physical connectivity, their BOLD signal should be highly correlated over time. Like Eguiluz et al. [11], we have chosen the Pearson correlation coefficient $r(i, j)$ to estimate the amount of activity correlation for each pair of voxels $(i, j)$ :

$$
r(i, j)=\frac{\langle V(i, t) V(j, t)\rangle-\langle V(i, t)\rangle\langle V(j, t)\rangle}{\sigma(V(i)) \sigma(V(j))},
$$

where $V(m, t)$ is the BOLD activity of the $m$-th voxel at time $t,\langle$.$\rangle denotes the time$ average, and $\sigma^{2}(V(m))=\left\langle V^{2}(m, t)\right\rangle-\langle V(m, t)\rangle^{2}$. An edge between the pair of voxel nodes is established if $|r(i, j)|>\theta$, where $\theta$ is a given correlation threshold (see Fig. 1). We have chosen an absolute value of correlation to derive a functional link between voxels. This means that both strongly positively and strongly negatively correlated voxels are included in the functional network. Because the correlation $r(i, j)$ is a symmetric function, the networks are undirected. After applying the correlation threshold $\theta$, we treat the networks as undirected and binary, i.e the edge either exist or not.

\section{Results}

\subsection{Whole brain functional networks - data analysis}

Here, the basic analysis of functional brain networks is presented for the three groups of participants. Particular three correlation thresholds for which the functional networks were derived were: $\theta_{1}=0.819398, \theta_{2}=0.899876$ and $\theta_{3}=$ 0.962249. Justification: For these whole brain functional networks, McCarthy et al. $[23,24]$ calculated average properties, like the number of nodes, degree, density, small world index, clustering coefficient, shortest path, local and global efficiency, and assortativity. These average characteristics were statistically compared in order to look for differences between the three groups of participants. Statistical null hypothesis consisted of no difference, for any functional network characteristics, between each pair of groups. McCarthy et al. [23, 24] have estimated that the statistically significant differences between average network measures at the $\alpha=10^{-2}$ level occurred for the functional networks created with the threshold $\theta_{1}=0.819398$, at the $\alpha=10^{-3}$ level for $\theta_{2}=0.899876$, and at the $\alpha=10^{-5}$ level for $\theta_{3}=0.962249$. We have decided to use the networks extracted by McCarthy et al. $[23,24]$ at the same values of thresholds for the inference of our models too.

Fig. 2 shows degree distributions of the HY functional brain networks for all correlation thresholds. For the lowest correlation threshold $\theta_{1}=0.819398$, the average number of nodes is $N_{1}^{H Y}=10487$, with the individual values lying in the 
Markošová M. et al.: Network models for changing degree distributions of...

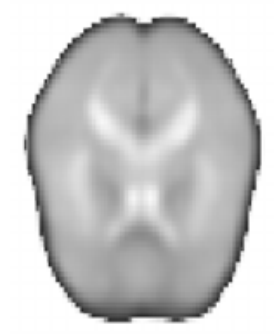

A

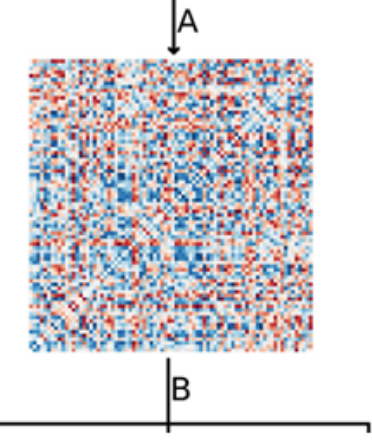

$\theta_{1}$

$\theta_{2}$
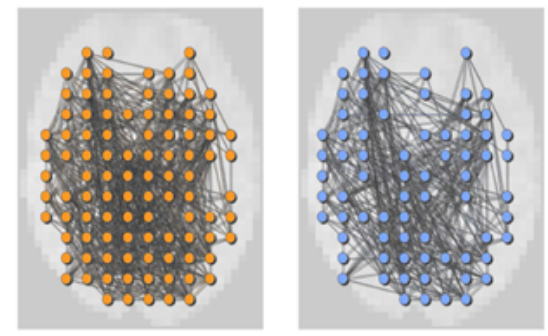

$\theta_{3}$

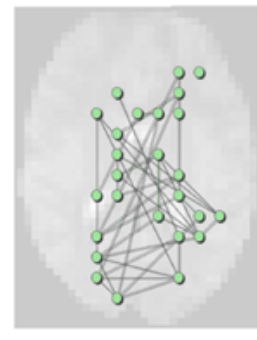

Threshold $\theta$

Fig. 1 Stages of creation of the functional brain network. A: Based on a chosen voxel activity correlation measure, a correlation matrix was calculated. This was done for the fMRI volume of each participant. B: Three unweighted, undirected networks were extracted from each correlation matrix, using three different correlation thresholds $\theta_{1}<\theta_{2}<\theta_{3}$. Based on which correlations crossed the given correlation threshold in the matrix of correlations, the resulting networks contain different number of nodes and edges.

interval $[9850,11140]$. As we can see in Fig. 2, for $\theta_{1}$, the tail of the distribution is too short in order to estimate the power law scaling exponent correctly. Thus, we conclude that the degree distribution for the lowest correlation threshold $\theta_{1}$, the functional brain networks are not scale-free because they do not have a power law character. 

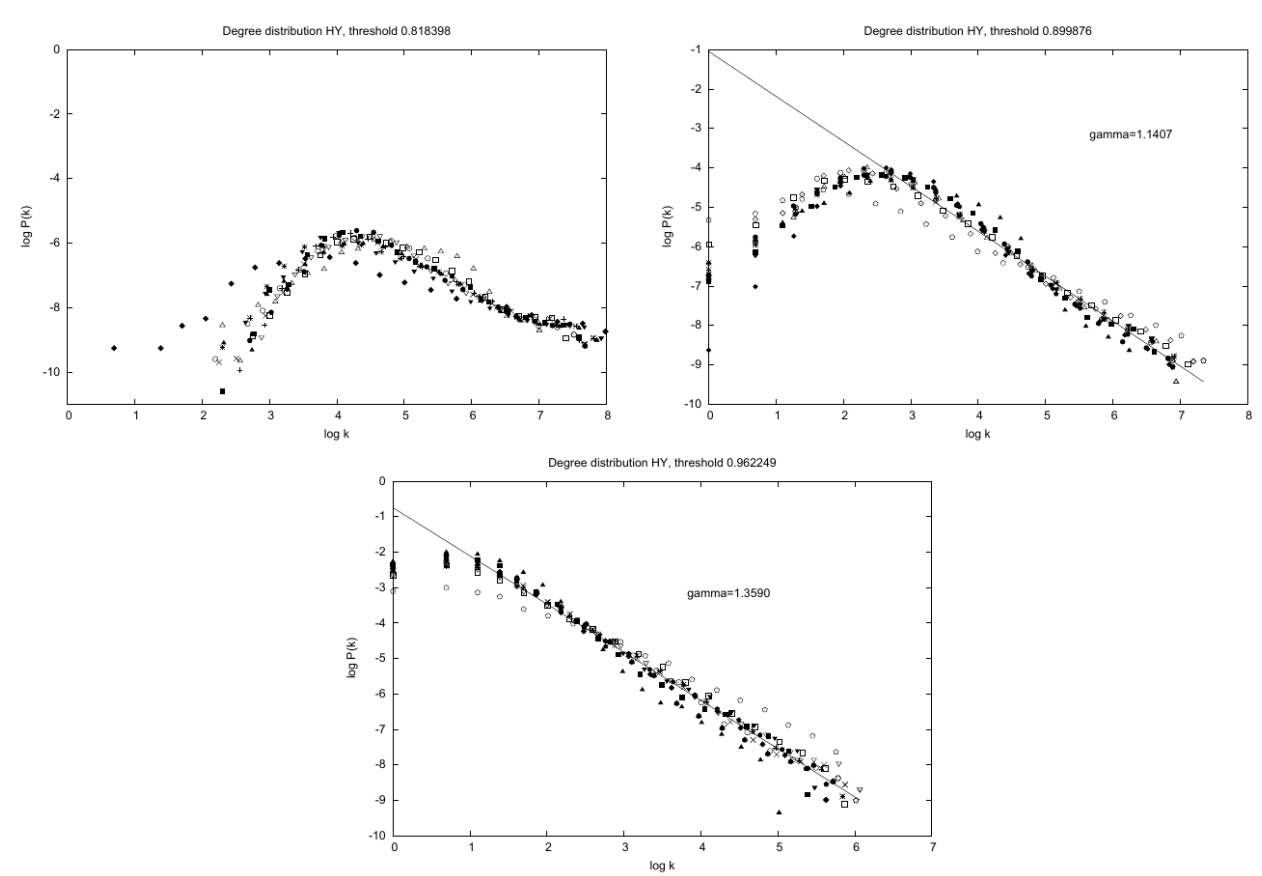

Fig. $2 H Y$ group. Degree distributions of the functional brain networks of participants from left to right: the lowest correlation threshold $\theta_{1}=0.818398$, the medium threshold $\theta_{2}=0.899876$, and the highest threshold $\theta_{3}=0.962249$. The figure shows that the scale-free character of the networks at the highest threshold is gradually being destroyed as $\theta$ gets lower and lower.

For the medium correlation threshold $\theta_{2}=0.899876$, the average number of nodes in the functional brain network is somewhat lower, i.e. $N_{2}^{H Y}=10426$. All individual networks have number of nodes in the interval [9842,11140]. As we can see, there is a pronounced power law tail for the medium correlation threshold, with the average scaling exponent $\gamma_{2}^{H Y}=1.14$. The scaling exponent is calculated as an average of all scaling exponents of all individual distributions.

For the highest correlation threshold $\theta_{3}=0.962249$, the average number of nodes in the functional brain network is the lowest, i.e. $N_{3}^{H Y}=7280$. Individual values are in the interval $[6482,8582]$. At the highest correlation threshold, the situation changes dramatically. All the functional brain networks have a power law degree distribution with the individual values of $\gamma_{3}^{H Y}$ in the interval [0.9346, 1.7812]. They have a well defined scale-free structure, with the average scaling exponent $\gamma_{3}^{H Y}=1.36$.

Next, we depict the degree distributions at the three correlation thresholds for the HE group (Fig. 3). For the lowest correlation threshold $\theta_{1}$, the average number of nodes was $N_{1}^{H E}=9486$. The individual values lie in the interval [8458,10179]. The tail of distribution is too short to estimate the power law scaling exponents correctly. Thus, we conclude that the functional brain networks for $\theta_{1}$ are not scale- 

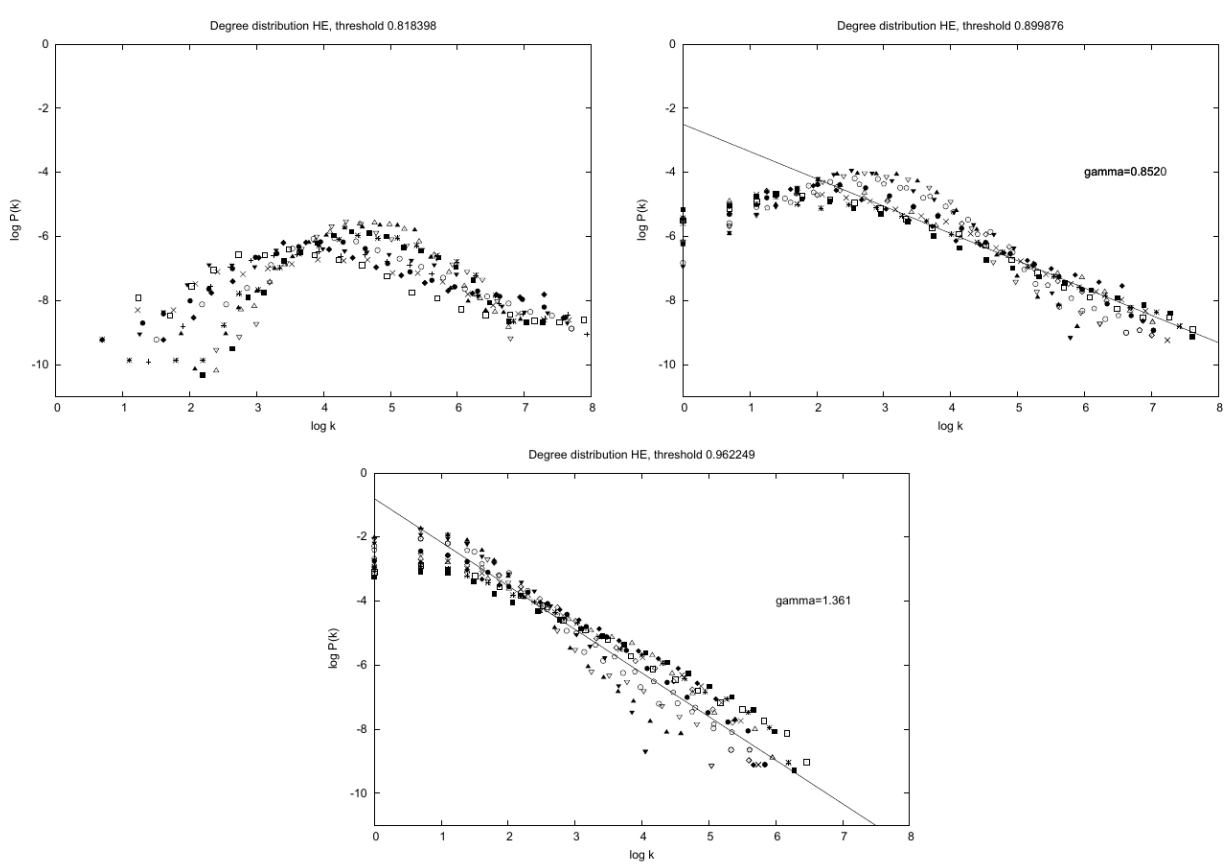

Fig. 3 HE group. Degree distributions of the functional brain networks of participants from left to right: the lowest correlation threshold $\theta_{1}=0.818398$, the medium threshold $\theta_{2}=0.899876$, and the highest threshold $\theta_{3}=0.962249$. The figure shows that the scale-free character of the networks at the highest threshold is gradually being destroyed as $\theta$ gets lower and lower.

free, because they do not have a power law character. For the medium threshold $\theta_{2}$, the average number of nodes is $N_{2}^{H E}=9467$. The number of nodes for the individual network lies in between the values $[8420,10156]$. The degree distribution for $\theta_{2}$ reveals more pronounced tail in the $\log -\log$ plots. However, it also shows more variability between individuals than the $\theta_{2}$ degree distribution of the HY group. Five individuals do not have the power law tail long enough to find a scaling exponent correctly. Therefore, they were not included in calculating the average $\gamma_{2}^{H E}=2.27$ exponent. For $\theta_{3}$, the average number of nodes is $N_{3}^{H E}=7034$ and the number of nodes is in the interval [5280,8382]. Analysis of the networks generated for the highest threshold $\theta_{3}$ reveals their scale-free character. The average scaling exponent $\gamma_{3}^{H E}=1.3609$. All the individual scaling exponents lying in the interval $[1.0500,2.0396]$. However, also for $\theta_{3}$, there is a higher variability among $\mathrm{HE}$ individual networks than in the HY group.

Finally, we analyzed the functional brain networks of the AE group (i.e. elderly participants with diagnosed mild or very mild Alzheimer's disease) for all three thresholds (Fig. 4). We would like to point out even greater individual variability of degree distributions for all three thresholds compared to the first two groups, namely HY and HE. Our analysis shows that even not all of the networks have a 

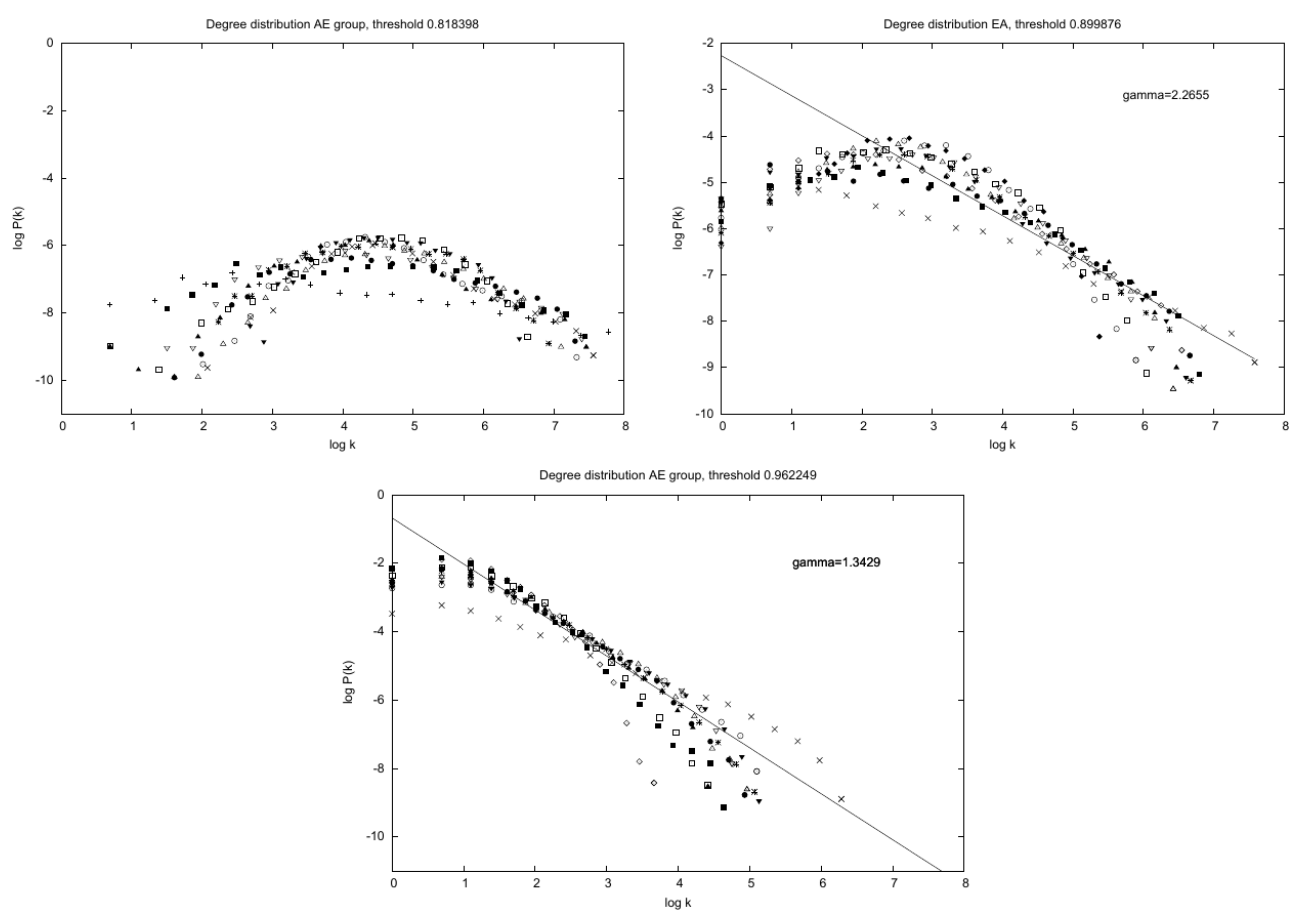

Fig. 4 AE group. Degree distributions of the functional brain networks of participants from left to right: the lowest correlation threshold $\theta_{1}=0.818398$, the medium threshold $\theta_{2}=0.899876$, and the highest threshold $\theta_{3}=0.962249$. The figure shows that the scale-free character of the networks at the highest threshold is gradually being destroyed as $\theta$ gets lower and lower.

scale-free property for the medium and highest thresholds. Here are the numerical results: For the lowest correlation threshold $\theta_{1}$, the networks are not scale-free. The average number of nodes in the group is $N_{1}^{A E}=8956$, the interval is [7825, 10204]. For the medium threshold $\theta_{2}$ threshold the average number of nodes is $N_{2}^{A E}=$ 8905 , having the individual values in the interval $[7825,10204]$. The average group $\gamma_{2}^{A E}=0.8641$ and the individual values lie in the interval $[0.5313,1.1520]$. Four out of 12 individual distributions have no power law tail and thus were excluded from the calculation. At the highest correlation threshold $\theta_{3}$, the average number of nodes is $N_{3}^{A E}=6679$ with the individual values in the interval [5575, 8394]. The degree distribution of the majority of networks has a power law character. The exception is just one outlier. The average scaling exponent is $\gamma_{3}^{A E}=1.3429$ (interval [0.8046, 2.1867]) (Fig. 4).

The results of degree distribution analysis are qualitatively similar for all groups of participants. The scale-free network structure at the highest threshold is gradually being destroyed as the correlation threshold $\theta$ gets lower and lower. In addition, compared to those of the HY group, the data of HE and AE groups are far less coherent due to a large individual variability between participants in these aged groups. 


\section{Models of changing degree distributions}

Our data analysis described in the previous section has revealed that the character of the functional brain networks depends on the value of correlation threshold. In particular, when the correlation threshold is the lowest, the degree distributions are not scale-free, but rather have a characteristics of the random graph. As the correlation threshold increases, the degree distributions gradually develop more and more pronounced power law tails (see Figs. 2, 3 and 4). Thus, we are interested in developing the models that explain the changing degree distribution of the functional brain networks with the correlation threshold and not time being an independent variable.

In the following analysis we are inspired by the work of Scholz et al. [30], who developed a model for the scale-free networks in which the noise increases and the degree distribution changes. The authors started from a scale-free network. The number of nodes was fixed to $N_{0}$, and the initial number of edges to $L_{0}$. Then the network was disturbed by some type of noise reflected by random link removal, random link exchange and random link addition. The authors have developed a model how the degree distribution drifts from the power law character to randomness with increasing the noise (randomness applied to edges) in the network over time.

However our goal is to explain the changes in degree distribution depending on the value of correlation threshold. For this purpose we assume the noise is affecting the degree distribution when the threshold of correlations between the voxels changes its value. Unlike the original model of Scholz et al. [30], in addition to changes in the number of edges, we assume an increase in the number of nodes as the threshold of correlation decreases. First, let us suppose, we lower the threshold step by step such that at each iteration corresponding to a small threshold change, one new node comes to the system. Thus, each step or iteration is marked by addition of a new node.

In the following equations, $n$ denotes the number of network growth steps. Let us first look at the effect of the random link addition as described by Scholz et al. [30]. The impact of random link addition is described by this rate equation:

$$
P(k, n+1)=\frac{2}{N_{0}} P(k-1, n)\left(1-\delta_{k, 0}\right)+\left(1-\frac{2}{N_{0}}\right) P(k, n) .
$$

$P(k, n)$ is the normalized number of nodes having the degree $k$ at the iteration $n$. $N_{0}$ denotes the initial number of nodes and $\delta_{k, 0}$ is the Kronecker delta function. The first term is a gain term; each end of the new edge can be added with the same probability to each of $N_{0}$ nodes. The second term is a loss term. Number 2 in nominator means, that each added link has two ends. Each edge end chooses randomly between available nodes. The asymptotic degree distribution for the latter rate equation is $P(k, n)=A(k, n) k^{-\gamma(k, n)}$, where $k$ denotes the degree and $n$ stands for the iteration. The analytical solution of this equation shows, that the scaling exponent $\gamma$ changes as (Scholz et al., 2005):

$$
\gamma(k, n)=\gamma_{0} e^{\left(\frac{2 n}{N_{0} k}\right)} .
$$


Based on this, we assume that lowering the correlation threshold corresponds to increasing the probability of addition random edges between the nodes in functional network. This in turn causes that the degree distribution looses its power law character. We have utilized this insight and developed two different yet related models based on the data and assumption of increasing the noise which affects not only the number edges but also the number of nodes as the correlation threshold is lowered.

Thus, we develop our model as follows. We start at the highest threshold $\theta_{3}$, at which the network is scale-free and has $N_{0}$ nodes, $L_{0}$ edges and the power law degree distribution. Then the threshold is lowered step by step. At each iteration step in lowering the correlation threshold, new nodes and edges are added to the network by both the preferential and random linking. In our model, a new node is added to the network when its signal is now correlated with any node (voxel) already present in the network, provided they were not previously correlated at the higher threshold to avoid duplicity. We assume, that each threshold's discrete and infinitesimally small change is accompanied by only one new node and on average the same number of new edges per iteration. In addition, each new node brings $a_{1}$ new edges that are linked preferentially and $a$ new edges that are linked randomly. Thus at each iteration, the total amount of edges brought by one node is $a+a_{1}$. This sum should be at least one, otherwise the node is not connected. At the same time, we assume that yet another process takes place. Namely, as the threshold decreases, some correlations between the pairs of nodes already present in the network become significant because now their value crosses a new value of the threshold. Therefore new edges are distributed preferentially $\left(b_{1}\right)$ or randomly (b) among the nodes beeing already in the network at the previous value of the higher threshold.

Thus, unlike the previous model (Eq. 4), both the number of nodes and edges in our network model increase. We created two models, let us call them the model A and the model B, which differ in certain details and are described below. First, we start with the network at the highest threshold with $N_{0}$ nodes, which is scale-free. As the threshold lowers, new nodes and correlations (edges) emerge.

The rate equation describing the above mentioned dynamic processes in both models is:

$$
P(k, n+1)=p_{k, k-1}(n) P(k-1, n)+\left(1-p_{k+1, k}(n)\right) P(k, n) .
$$

In the model $\mathrm{A}$, the transition term $p_{k, k-1}(n)$ reads:

$$
p_{k, k-1}(n)=\frac{a+2 b}{N_{0}+n}+\frac{\left(a_{1}+2 b_{1}\right)(k-1)}{2 L_{0}+A(n)},
$$

where $A(n)=2\left(a+b+a_{1}+b_{1}\right) n$.

In Eq. (6), $P(k, n)$ is the normalized number of nodes having the degree $k$ at iteration $n$. This number changes, due to the fact that some nodes having at the previous iteration $n$ the degree $k-1$, gain a new edge. This is expressed in the first term of the Eq. (6). The second term expresses the situation that for some nodes having the degree $k$ at iteration $n$ with the probability $1-p_{k+1, k}(n)$, no new edges are added. In Eq. (7), $N_{0}, L_{0}$ denote initial number of nodes and edges, $a, b$ are the number of randomly added edges per iteration, where $a$ is the number of 
edges fetched by a new-coming node and $b$ is the number of edges added between older network nodes. Similarly, $a_{1}$ denotes the number of edges by which a new node links preferentially to the network and $b_{1}$ is the number of edges linking older nodes preferentially. Factor two at the coefficients denotes, that these edges are linked by both of their ends, unlike to those edges, which have just one edge end linked to the new-coming node. The transition term $p_{k+1, k}(n)$ describes how the number of nodes having the degree $k$ changes due to the above mentioned dynamic processes. The first term of $p_{k+1, k}(n)$ says that $(a+2 b)$ edge ends are linked randomly, with equal probability $\frac{1}{N_{0}+n}$ to the node in the network. The number of nodes in the network at iteration $n$ is $N_{0}+n$, because as stated before, at each iteration (threshold step) exactly one new node appears. The second term of $p_{k+1, k}(n)$ describes preferential attachment of $\left(a_{1}+2 b_{1}\right)$ edge ends with the probability proportional to the node degree. The normalization factor is the sum of all node degrees $2 L_{0}+A(n)$. The first term of the Eq. (6) is a gain term and the second one is a loss term. In the model A, we neglect what happens with the other links. In other words, we only pay attention to the fact how link addition affects the degree $k$ (Eqs. 6, 7) .

We also created a more refined model $\mathrm{B}$, in which we do care about what happens to the other links. If a link is added to a certain $k$-degree node, the other links have to attach elsewhere.

Eq. (6) is still valid in the model $\mathrm{B}$, but the $p_{k, k-1}(n)$ term is different:

$$
\begin{aligned}
& p_{k, k-1}(n)=\frac{a+2 b}{N_{0}+n}\left(\frac{N_{0}+n-1}{N_{0}+n}\right)^{a+2 b-1}\left(\frac{2 L_{0}+A(n)-(k-1)}{2 L_{0}+A(n)}\right)^{a_{1}+2 b_{1}}, \\
& +\frac{\left(a_{1}+2 b_{1}\right)(k-1)}{2 L_{0}+A(n)}\left(\frac{N_{0}+n-1}{N_{0}+n}\right)^{a+2 b}\left(\frac{2 L_{0}+A(n)-(k-1)}{2 L_{0}+A(n)}\right)^{a_{1}+2 b_{1}-1} .
\end{aligned}
$$

Here also $A(n)=2\left(a+b+a_{1}+b_{1}\right) n$ and the other terms denote the same as previous.

This model describes the probability of the situation, in which exactly one edge links by the defined manner (either randomly or preferentially) to a node having the degree $k$, while other edges in this model cannot attach to any $k$-degree node, but somewhere else to the nodes with different degree. This might be seen as a first step of model refinement. The second one could be to allow one or two edges to link to the node with the degree $k$ and the other edges linking elsewhere. We leave this and other possible variants to the discussion.

\section{Results of numerical simulations}

We simulated both models A and B (Eqs. 7, 8) numerically. Simulations were done for the average functional brain networks from the HY, HE and AE group of participants with respect to the thresholds $\theta_{2}$ and $\theta_{1}$. The results for all groups are presented in Figs. 5-10. First, we used the experimental data to find the parameters of initial power law degree distribution at the highest threshold $\theta_{3}$ (Eq. 9) corresponding to the initial number of iterations $n_{0}=0$, i.e. 

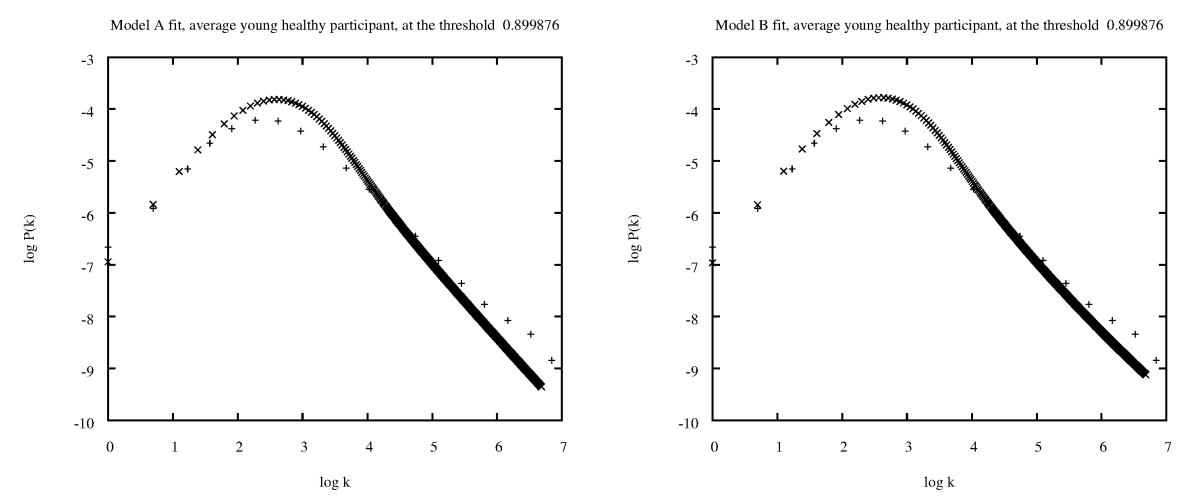

Fig. $5 \mathrm{HY}$, fit at the threshold $\theta_{2}$. Left: Model A fit for the average functional brain network, '+' - data, ' $x$ ' - simulation. 800 iterations, parameters $a=0.0259$, $a_{1}=1.7545, b=5.3677, b_{1}=286.0118, M S E=0.4059$. Right: Model $B$ fit for the same average functional brain network, ' $x$ ' - data, '+' - simulation. 400 iterations, parameters $a=0.0004, a_{1}=9.9534, b=5.4668, b_{1}=277.7394, M S E=0.3366$.
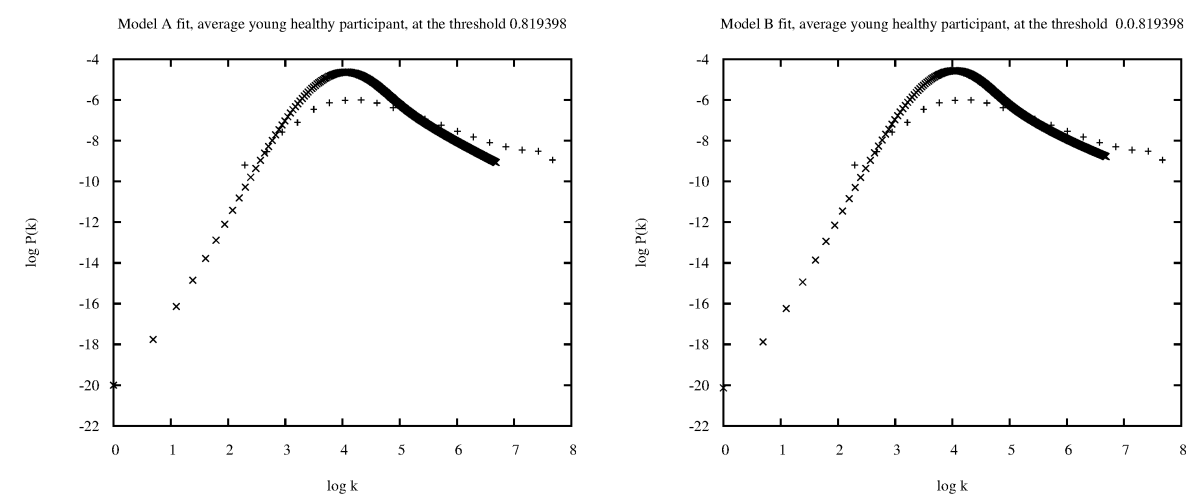

Fig. $6 \mathrm{HY}$, fit at the threshold $\theta_{1}$. Left: Model A fit for the average functional brain network, '+' - data, ' $x$ ' - simulation. 800 iterations, parameters $a=0.01628$, $a_{1}=3.4091, b=22.3423, b_{1}=546.9023, M S E=0.9642$. Right: Model $B$ fit for the same average functional brain network, ' $x$ ' - data, ' + ' - simulation. 400 iterations, parameters $a=6.0300, a_{1}=0.6902, b=19.6874, b_{1}=546.2624$, $M S E=0.8563$.

$$
P(k)=c k^{-\gamma}
$$

Both parameters $c$ and $\gamma$ were derived from the data. Next, the power law distribution function $P(k)$ (Eq. 9) has been normalized by the constant $z$ based on the data and calculated from the equation

$$
z=\int_{1}^{\infty} P(k) d k,
$$


Markošová M. et al.: Network models for changing degree distributions of. .

Model A fit, average elderly healthy participant, at the threshold 0.899876

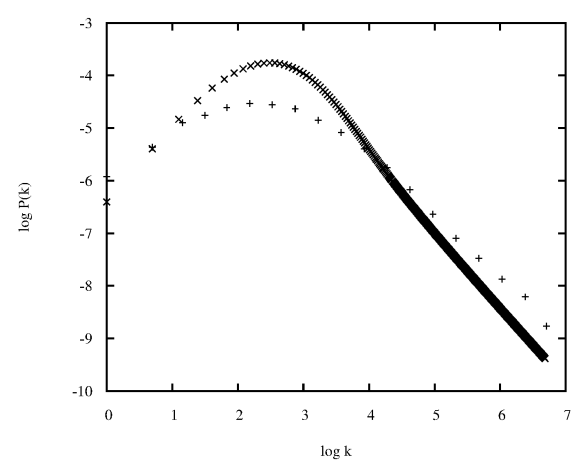

Model B fit, average elderly healthy participant, at the threshold 0.899876

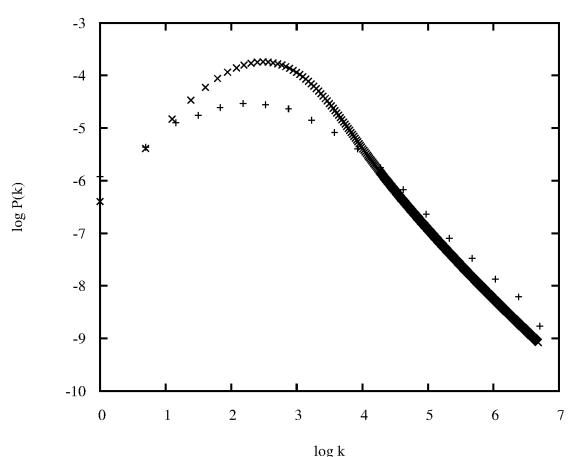

Fig. $7 \mathrm{HE}$, fit at the threshold $\theta_{2}$. Left: Model A fit for the average functional brain network, '+' - data, ' $x$ ' - simulation. 800 iterations, parameters $a=0.0012$, $a_{1}=6.8472, b=5.6091, b_{1}=435.3524, M S E=0.5211$. Right: Model $B$ fit for the same average functional brain network, ' $x$ ' - data, ' + ' - simulation. 400 iterations, parameters $a=0.0393, a_{1}=17.1701, b=5.7155, b_{1}=424.8850, M S E=0.4693$.
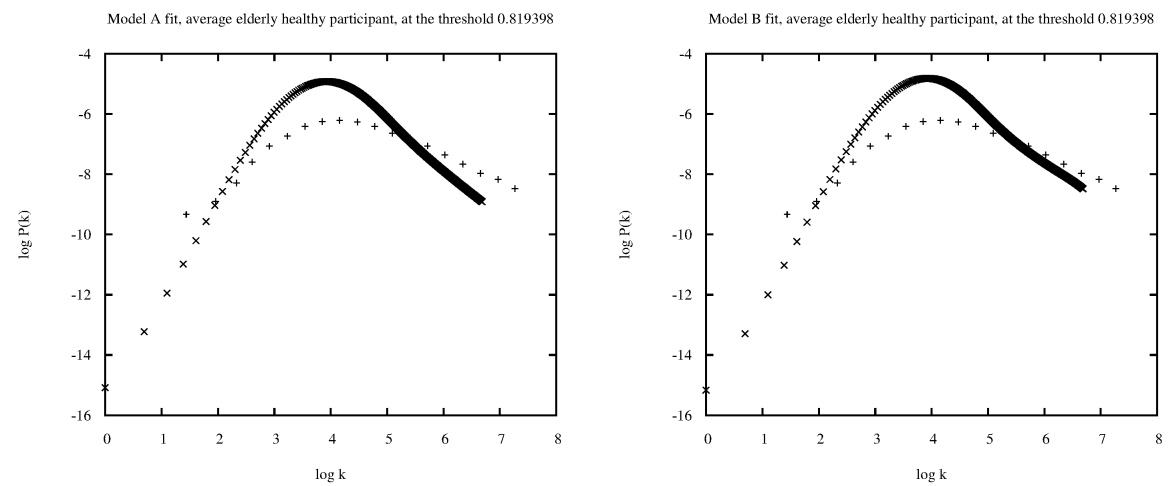

Fig. $8 \mathrm{HE}$, fit at the threshold $\theta_{1}$. Left: Model A fit for the average functional brain network, ' + ' - data, ' $x$ ' - simulation. 800 iterations, parameters $a=0.0453$, $a_{1}=0.9549, b=18.1390, b_{1}=1439.9307, M S E=0.9544$. Right: Model $B$ fit for the same average functional brain network, ' $x$ ' - data, ' + ' - simulation. 400 iterations, parameters $a=0.9991, a_{1}=0.0060, b=17.9520, b_{1}=1440.1210$, $M S E=0.8975$.

so that the sum of all probabilities of the initial distribution is close to one after the normalization. We excluded the networks, for which the integral (Eq. 10) does not converge. In fact, there was just one case in the HY group. 

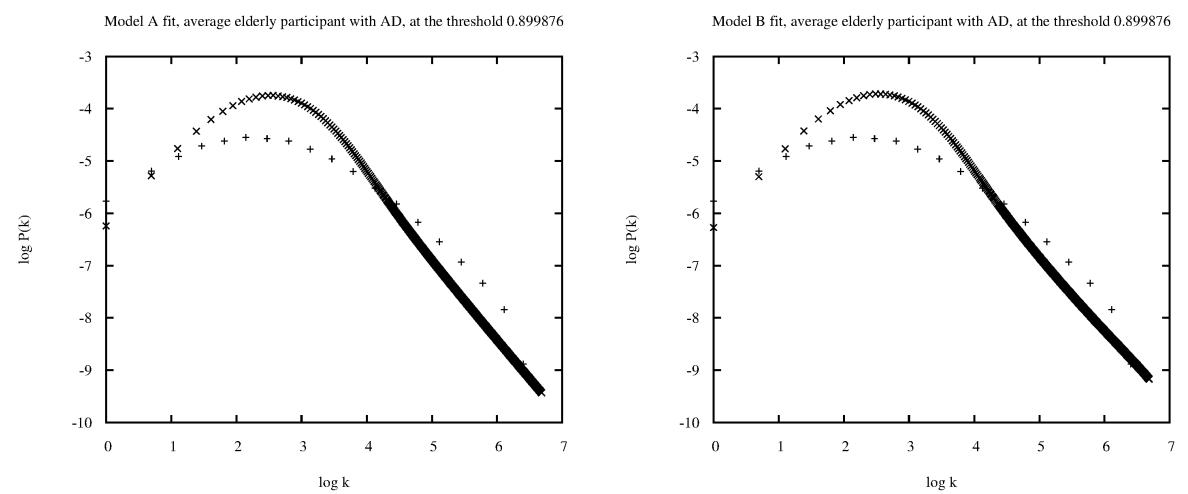

Fig. $9 \mathrm{AE}$, fit at the threshold $\theta_{2}$. Left: Model $A$ fit for the average functional brain network, ' + ' - data, ' $x$ ' - simulation. 800 iterations, parameters $a=0.0475$, $a_{1}=0.9529, b=5.405, b_{1}=236.9792, M S E=0.5374$. Right: Model $B$ fit for the same average functional brain network, ' $x$ ' -data, ' + ' - simulation. 400 iterations, parameters $a=0.0018, a_{1}=3.3738, b=5.5351, b_{1}=234.4743, M S E=0.5188$.
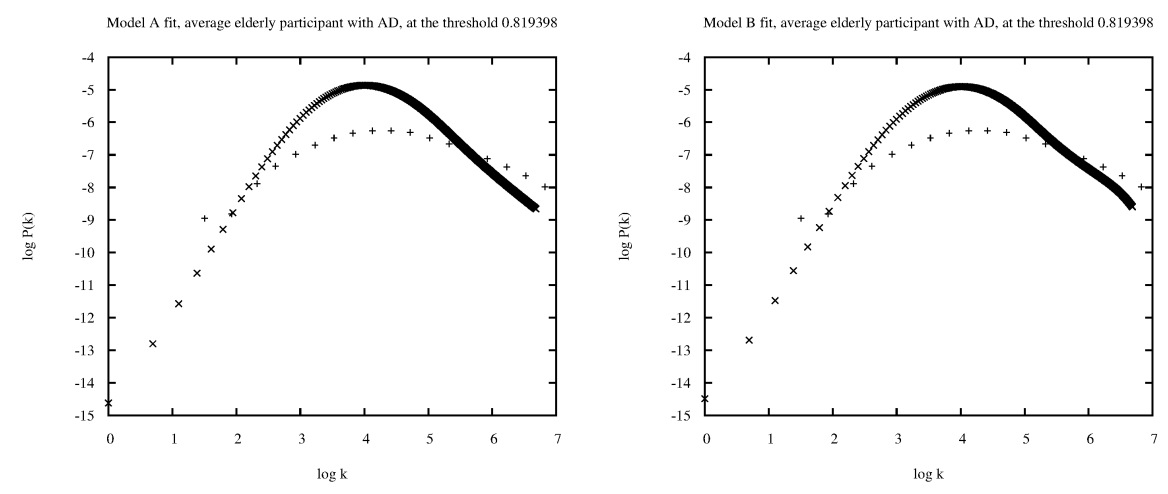

Fig. 10 AE, fit at the threshold $\theta_{1}$. Left: Model A fit for the average functional brain network, ' + ' - data, ' $x$ ' - simulation. 800 iterations, parameters $a=0.0061$, $a_{1}=2.0289, b=17.8595, b_{1}=929.5204, M S E=0.9189$. Right: Model $B$ fit for the same average functional brain network, ' $x$ ' - data, ' + ' - simulation. 400 iterations, parameters $a=0.0121, a_{1}=0.0025, b=17.4500, b_{1}=931.9510$, $M S E=0.8933$.

In our simulations, we first used the models A and B to simulate the change of degree distribution from the highest correlation threshold $\theta_{3}$ to the medium threshold $\theta_{2}$. Each model has been iterated $N_{2}-N_{0}$ times, where $N_{0}, N_{2}$ denote the number of nodes for the threshold $\theta_{3}$ and at the the correlation threshold $\theta_{2}$, respectively. In each network growth step (a discrete small threshold change and addition of one node) a fixed number of edges is added, namely $\frac{\left(L_{2}-L_{0}\right)}{\left(N_{2}-N_{0}\right)}$, where $L_{2}$ is the number of network edges obtained from the measured data at the threshold $\theta_{2}$ and $L_{0}$ is the initial number of edges. To find the best values for the set of 
parameters $a, b, a_{1}, b_{1}$ we used the hill climbing algorithm. The mean square error (MSE) between the measured and simulated data has been calculated as an optimization function. Each hill climbing simulation started from seven different initial values. Then, from the best fit parameters, fifteen new sets of parameter values were derived by slight perturbations of the currently best fit parameter set. This is a standard procedure in the hill climbing optimization algorithm. The hill climbing algorithm was iterated 800 times for the model A, while only 400 times for the model B were necessary. Next, we did the same optimization as before to account for the data at the lowest threshold $\theta_{1}$. The hill climbing algorithm was iterated $N_{1}-N_{0}$ times, where $N_{1}$ is the number of nodes at the lowest threshold $\theta_{1}$. Consequently, the number of edges added in each threshold (network growth) step is equal to $\frac{\left(L_{1}-L_{0}\right)}{\left(N_{1}-N_{0}\right)}$, where $L_{1}$ is the number of edges at the threshold $\theta_{1}$.

\section{Summary and discussion}

We analyzed the characteristics of degree distributions of functional brain networks which were created for the three different correlation thresholds of the activity correlation between each pair of voxels. Functional brain networks were extracted from the fMRI data obtained for the HY (healthy young), HE (healthy elderly) and AE (elderly with Alzheimer disease) participants. We have found that:

- At the highest correlation threshold $\theta_{3}$ the degree distributions can be considered as scale-free with developed power law tails (Figs. 2, 3, 4). This is especially well seen for the degree distributions in the HY group (Fig. 2). This result corresponds to previous studies done on different fMRI data sets [11,20].

- In general, the character of the degree distribution of the functional brain networks changes depending on the value of correlation threshold of pairwise voxel activity. As the correlation threshold decreases, the power law tails in the degree distributions are less and less pronounced. Thus, the functional brain network looses its scale-free structure as can be seen in Fig. 2, Fig. 3, Fig. 4.

- There are individual differences in the functional brain network degree distributions in each of the three groups of participants (HY, HE, AE group), while the HY group exhibits the smallest individual differences thus is the most coherent (Fig. 2) compared to the $\mathrm{HE}$ and $\mathrm{AE}$ groups (see Fig. 3 and 4). Nevertheless, the degree distributions in all of the three groups change in a similar way with the changing correlation threshold.

- To explain the degree distribution changes with the decreasing threshold, we created two models A and B, while the model B is a refinement of model A. The crucial factor both models contain is an increasing noise as the activity correlation threshold is lowered, which in our models corresponds to an increase of the number of random correlations between the voxels. In all simulations model B gives better results then the model A, based on MSE. 
- Once the parameters $a_{1}, a, b_{1}, b$ are established, the models enable one to predict, how the degree distributions will look at the intermediate correlation thresholds, for which we do not have the numerical data.

The main contribution of our work is creation of novel mathematical network models developed to address the question which network wiring processes can cause the scale-free functional brain networks to loose the their scale-free topology. Both models describe the dynamic processes that occur in the growing, initially scale-free network. Increasing the noise in both models is reflected by the random distribution of a constant number of edges among the nodes being already present in the network. On the other hand, the preferentially distributed edges among the nodes already in the network, still support the scale-free structure. At the same time, the network also grows by the node addition, while each node brings a constant number of new edges, which are distributed either preferentially or randomly.

Both models reflect a specific view on the data, in which the role of independent variable is played by changing the threshold. The threshold (network growth) steps are adjusted to allow exactly one new node to appear in the network, as described previously. We started with the scale-free degree distributions at the highest threshold $\theta_{3}$. The parameters $c$ and $\gamma$ of the degree distribution are derived from the data at the highest threshold (Eq. 9). Then, we simulated both models numerically, optimizing their parameters to fit the data at lower thresholds $\theta_{2}$ and $\theta_{1}$.

In general we can state that in the group of young healthy participants the model $\mathrm{B}$, which is a refinement of the model A performs better. That means that the model $\mathrm{B}$ gives, in average, better fit to the data even for lover number of iteration steps, namely 400 (model B) versus 800 (model A). The quality of the fit is measured by the MSE. In the Appendix we present the best and the worst fits for the threshold $\theta_{1}$ and $\theta_{2}$, together with MSE.

We made a check of an averaged functional brain networks degree distributions in another groups of participants, namely $\mathrm{HE}$ and $\mathrm{AE}$, and we found that for the $\mathrm{HE}$ and AE group both models are qualitatively correct. Also here the model B gives better outcome. More research is necessary to explain individual quantitative differences in data fits. The question is, to which extend are these differences caused by the variable and noisy data sets and to which extend they are due to the processes not yet captured by our models. We leave these studies to the future research.

As we have stated before, in real biological data it is difficult to derive accurate conclusions, because the data themselves are riddled with noise arising from experimental measurement. In addition, there is also variability arising from individual brain differences, which seems to increase with the age of participants and with the onset of the brain disease. We therefore mostly speak about tendencies. We get better fits at the correlation threshold $\theta_{2}$ in comparison to the data fits at the lowest threshold $\theta_{1}$. This indicates, that the simplification, that at each network growth (threshold) step a constant number of edges is distributed may not be completely valid. This can be derived from particular functional brain networks. It turns out that that the number of edges increases far more quickly between the thresholds $\theta_{2}$ and $\theta_{1}$ than between the thresholds $\theta_{3}$ and $\theta_{2}$. On the other hand, 
the difference in the number of nodes between $\theta_{2}$ and $\theta_{3}$ is measured in hundreds and only in units or tens between the thresholds $\theta_{2}$ and $\theta_{1}$.

These findings indicate, that the number of edges distributed at each network growth step (i.e. threshold change) is threshold dependent quantity, which should be taken into account in a future model. Also the possibility to measure each iteration by a newly appearing edge should bring another view to the dynamic processes. This also needs an estimation of the probability with which a node appears at a certain edge addition. Another possibility is to refine the existing models even more and allow at most two edges to link to a node with the degree $k$ and check, whether a new model improves the data match at the lower thresholds or not. If yes, we can check a model which allows at most three edges to link to a node with the degree $k$, etc. If not, then further model refinement, which of course enhances the model complexity will not be necessary. We leave this to the future investigation.

We created two mathematical models (A, B) of noisy growing networks inspired by the changes of degree distribution of functional brain networks derived from the fMRI brain scans of healthy young, healthy elderly participants and elderly participants with Alzheimer disease. Both models capture degree distribution changes at different correlation thresholds and fit the data well, both qualitatively and to a certain degree also quantitatively. This is remarkable given the data were collected from different age groups and also from the subjects with starting Alzheimer disease. In conclusion, we would like to point out, that our models show in general, what kind of local processes can destroy the initially scale-free structure in complex networks.

\section{Conflict of Interest Statement}

The authors declare that the research was conducted in the absence of any commercial or financial relationships that could be construed as a potential conflict of interest.

\section{Funding}

We acknowledge the financial support of the VEGA grant No. 1/0039/17. In addition, MM and LB gratefully acknowledge the financial and technical support of the Department of Computer Science, University of Otago, Dunedin, New Zealand, where the preliminary work was done.

\section{Acknowledgement}

We are grateful to Paul McCarthy for providing us with functional brain networks that we used as the basis for inference of dynamic models and also for individual picture parts used for Fig. 1. We are also thank Tatiana Jajcayová and Andrej Jursa for fruitful discussions.

\section{Appendix}

To demonstrate the robustness of our models, we numerically simulated both models A and B (Eqs. 7, 8) for individual networks in the HY group. Each simulation 
was compared to the experimental data data for the thresholds $\theta_{2}$ and $\theta_{1}$. In Figs. $11-15$, we present the individual best and the worst fits for the thresholds $\theta_{2}$ and $\theta_{1}$.
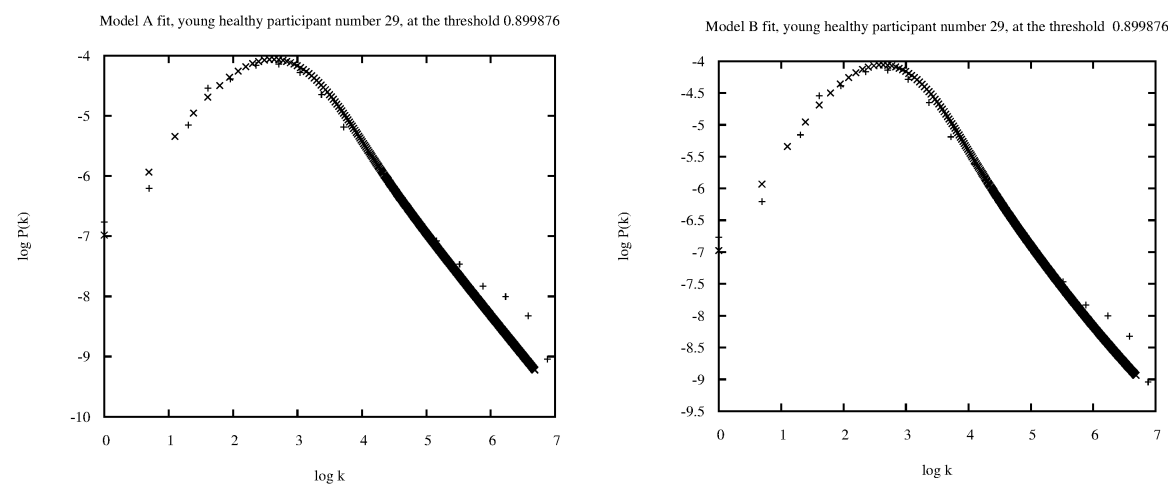

Fig. $11 H Y$, the best match for the threshold $\theta_{2}$. Left: Model $A$ for the functional brain network of the participant No. 29, '+' - data, ' $x$ ' - simulation. Number of iterations is 800, parameters $a=0.0148, a_{1}=3.58178, b=4.4640, b_{1}=240.1464$, $M S E=0.296$. Right: Model $B$ fit for the functional brain network of the same participant. ' $x$ ' - data, ' + ' - simulation. Number of iterations is 400 , parameters $a=0.0455, a_{1}=1.2881, b=4.4422, b_{1}=242.4312, M S E=0.1957$.
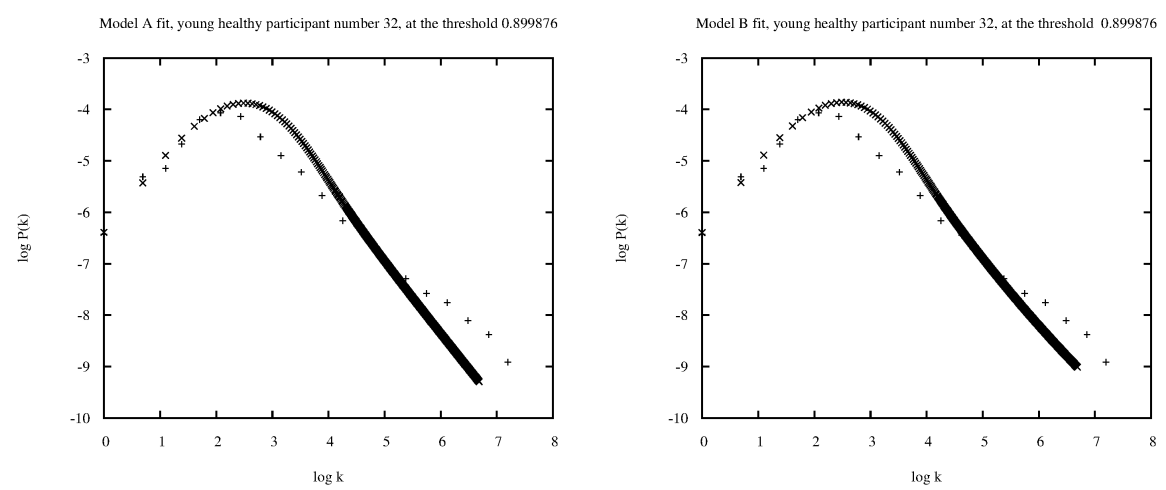

Fig. $12 H Y$, the worst match for the threshold $\theta_{2}$, , ' + ' - data, ' $x$ ' - simulation. Left: Model A for the functional brain network of the participant No. 32, 800 iterations, parameters $a=0.0209, a_{1}=4.8459, b=19.2407, b_{1}=456.7224$, $M S E=0.4554$. Right: Model $B$ fit for the functional brain network of the same participant, 400 iterations, parameters $a=0.0025, a_{1}=3.1801, b=4.7920, b_{1}=$ 472.8554, $M S E=0.4464$ 
Markošová M. et al.: Network models for changing degree distributions of. .

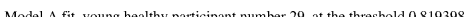

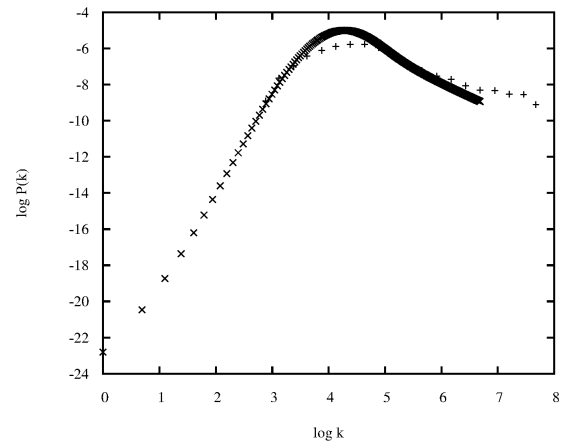

Model B fit young healthy particinant number 29 , a the threshold 0819398

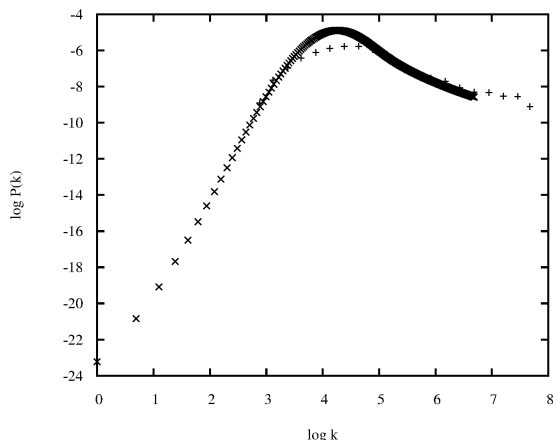

Fig. $13 H Y$, the best match for the threshold $\theta_{1}$. Left: Model A fit for the functional brain network of the young healthy participant No. 29, '+' - data, ' $x$ ' - simulation. 800 iterations, parameters $a=0.0359, a_{1}=0.9656, b=23.2207, b_{1}=517.2278$, $M S E=0.6814$. Right: Model $B$ fit for the functional brain network of the same participant, ' $x$ ' - data, ' + ' - simulation. 400 iterations, parameters $a=10.7086$, $a_{1}=1.1310, b=18.5728, b_{1}=511.0376, M S E=0.5028$.
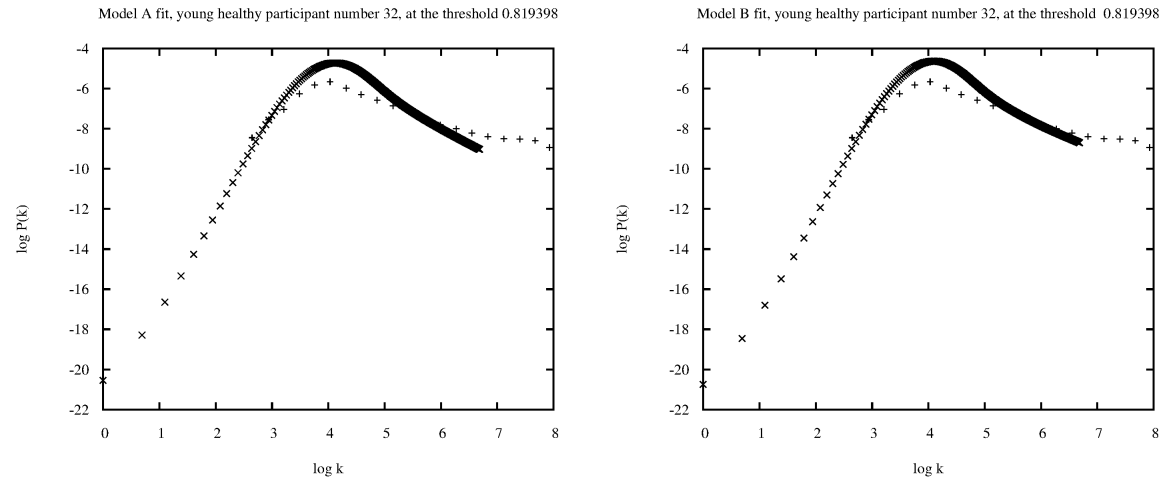

Fig. $14 H Y$, the match for the threshold $\theta_{1}$, participant 32. Left: Model $A$ fit for the functional brain network of the young healthy participant No. 32, '+' - data, ' $x$ ' - simulation. 800 iterations, parameters $a=0.7070, a_{1}=0.2957, b=24.7739$, $b_{1}=868.3084, M S E=0.9455$. Right: Model $B$ fit for the functional brain network of the same participant, ' $x$ ' - data, '+' - simulation. 400 iterations, parameters $a=6.1255, a_{1}=0.1614, b=22.6218, b_{1}=865.1763, M S E=0.76154$. 
Neural Network World 5/2020, 309-332
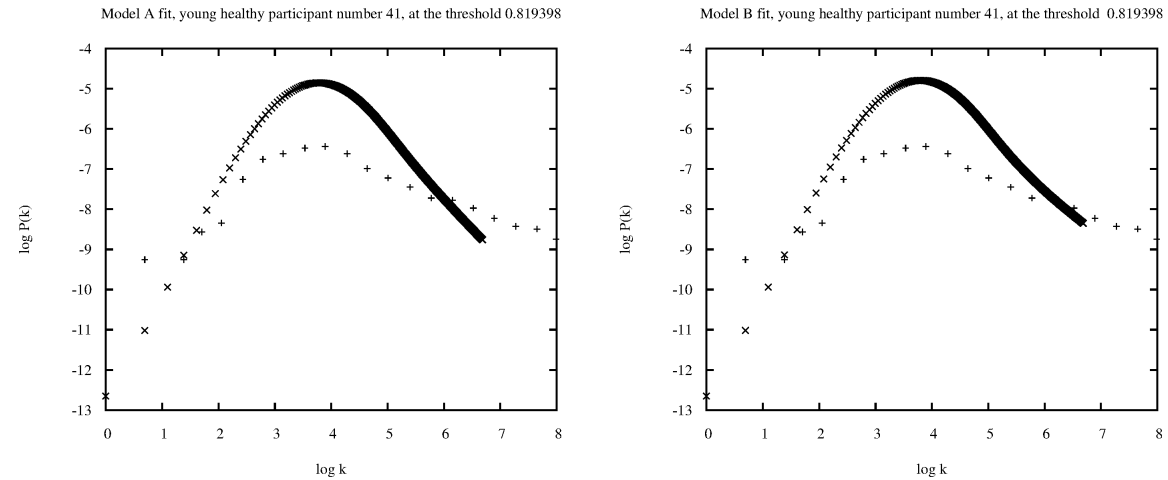

Fig. $15 H Y$, the worst match for the threshold $\theta_{1}$. Left: Model A fit for the functional brain network of the young healthy participant No. 41, '+' - data, ' $x$ ' - simulation. 800 iterations, parameters $a=0.0652, a_{1}=0.9377, b=10.4910$, $b_{1}=825.8862, M S E=1.1847$. Right: Model $B$ fit for the functional brain network of the same participant, ' $x$ ' - data, ' + ' - simulation. 400 iterations, parameters $a=0.0169, a_{1}=0.0078, b=10.5942, b_{1}=826.761, M S E=1.0848$.

\section{References}

[1] ACHARD S., SALVADOR R., WHITCHER B., SUCKLING J., BULLMORE E. A resilient, low-frequency, small-world human brain functional network with highly connected association cortical hubs. The Journal of Neuroscience, 2006, 26(1), pp. 63--72, doi: 10.1523/ JNEUROSCI . 3874-05.2006.

[2] ANDERSSON J.L., JENKINSON M., SMITH S. Non-linear registration, aka Spatial normalisation. FMRIB Centre, Oxford University, U.K., 2007. FMRIB Technical Report TR07JA2. Available from: https://www.fmrib.ox.ac.uk/datasets/techrep/tr07ja2/tr07ja2.pdf

[3] I BARABÁSI A.-L., ALBERT R. Emergence of scaling in random networks. Science, 1999, 286(5439), pp. 509-512, doi: 10.1126/science.286.5439.509.

[4] BASSETT D.S., BULlMORE E. Small-world brain networks. Neuroscientist, 2006, 12(6), pp. 512-523, doi: 10.1177/1073858406293182.

[5] BUCKNER R.L., SNYDER A. Z., SANDERS A.L., RAICHLE M.E., MORRIS J.C. Functional brain imaging of young, nondemented, and demented older adults. Journal of Cognitive Neuroscience 2000, 12 (Supplement 2), pp. 24-34, doi: 10.1162/089892900564046.

[6] BUCKNER R. L. Three principles for cognitive aging research. In: R. CABEZA, L. NYBERG, D. PARK, eds. Cognitive Neuroscience of Aging. Oxford University Press, 2005, pp. 267-285, doi: 10.1093/acprof : oso/9780195156744.003.0011.

[7] BUXTON R.B. Introduction to Functional Magnetic Resonance Imaging: Principles and Techniques (2nd edition), Cambridge U.K.: Cambridge Univ. Press, 2009.

[8] CIFTCI K. (2011). Minimum spanning tree reflects the alterations of the default mode network during Alzheimer's disease. Ann. Biomed. Eng. 2011, 39, pp. 1493-1504, doi: 10. 1007/s10439-011-0258-9.

[9] COX R.W. AFNI: What a long strange trip it's been. Neuroimage 2012, 62(2), pp. 743-747, doi: $10.1016 / j$.neuroimage 2011.08 .056 .

[10] DOROGOVTSEV S.N., MENDES J.F.F. Evolution of Networks. Oxford U.K.: Oxford University Press, 2003, doi: 10.1093/acprof : oso/9780198515906.001.0001. 
Markošová M. et al.: Network models for changing degree distributions of...

[11] EGUì LUZ V.M., CHIALVO D.R., CECCHI G.A., BALIKI M., APKARIAN A.V. Scale-free brain functional networks. Phys. Rev. Lett. 2005, 94, 018102, doi: 10.1103/PhysRevLett. 94. 018102.

[12] ERDÖS P., RÉNYI A. On the evolution of random graphs. Publ. Maths. Inst. Hung. Acad. Sci. 1960, 5, pp. 17-61.

[13] GREiCiUs M. D., SRIVASTAVA G., REISS A.L., MENON V. Default-mode network activity distinguishes Alzheimer's disease from healthy aging: evidence from functional MRI. Proc. Natl. Acad. Sci USA 2004, 101, pp. 4637-4642, doi: 10.1073/pnas. 0308627101.

[14] HAYASAKA S., LAURIENTI P.J. Comparison of characteristics between region- and voxelbased network analysis in resting-state fMRI data. Neuroimage 2010, 50, pp. 499-508, doi: $10.1016 / j$.neuroimage .2009 .12 .051$.

[15] HORWITZ B., McINTOSH A.R., HAXBY J.V., GRADY C.L. Network analysis of brain cognitive function using metabolic and blood flow data. Behavioural Brain Research 1995 , 66 (1-2), pp. 187-193, doi: 10.1016/0166-4328(94)00139-7.

[16] HUGhes C.P., BERG L., Danziger, W. L., Coben, L. A., and Martin, R. L. (1982). A new clinical scale for the staging of dementia. British Journal of Psychiatry 140 (6), 566-572. doi: $10.1192 /$ bjp.140.6.566.

[17] JENKINSON M., BANNISTER P., BRADY M., SMITH S. Improved optimization for the robust and accurate linear registration and motion correction of brain images. NeuroImage 2002, 17(2), pp. 825-841, doi: 10.1016/s1053-8119(02)91132-8.

[18] MANDELL A.M., GREEN R.C. "Alzheimer's disease". In: A.E. BUDSON, N.W. KOWALL, eds. The Handbook of Alzheimer's Disease and Other Dementias, eds. Wiley-Blackwell, 2011, pp. 3-91.

[19] MARKOŠOVÁ M. Network model of human language. Physica A: Statistical Mechanics and its Applications 2008, 387(2), pp. 661-666, doi: 10.1016/j.physa.2007.09.027.

[20] MARKOŠOVÁ M., FRANZ E.A., BENUSKOVA L. Topology of brain functional networks: towards the role of genes. In: M. KÖPPEN, N. KASABOV, G. COGHILL, eds. Advances in Neuro-Information Processing, Lecture Notes in Computer Science, Volume 5506, Berlin: Springer-Verlag, 2009, pp. 111-118, doi: 10.1007/978-3-642-02490-014.

[21] MARKOŠOVÁ M. Dynamic model of functional brain networks. ITAT 2016 CEUR Workshop Proceedings, Tatranské Matliare, 2016, 1649, pp. 226-231.

[22] MARKOŠOVÁ M. Network model inspired by the real data - functional brain networks. Proceedings of the 17th conference on Aplied mathematics APLIMAT 2018, Bratislava: Slovenská technická univerzita Press, 2018, pp. 733 - 743, doi: 10.3389/fnagi.2014.00301.

[23] McCARTHY P., BENUSKOVA L., FRANZ E.A. Functional network analysis of aging and Alzheimer's disease: Results. Dunedin: University of Otago, New Zealand, 2013. Technical Report OUCS-2013-12. Available from: http://www.cs.otago.ac.nz/research/publications/OUCS-2013-12.pdf

[24] McCARTHY P., BENUSKOVA L., FRANZ E.A. The age-related posterior-anterior shift as revealed by voxelwise analysis of functional brain networks. Frontiers in Aging Neuroscience 2014, 6:301, doi: 10.3389/fnagi.2014.00301.

[25] McINTOSH A.R., GONZALES-LIMA F. Structural equation modeling and its application to network analysis in functional brain imaging. Human Brain Mapping 1994, 2(2), pp. 2-22, doi: $10.1002 / \mathrm{hbm} .460020104$.

[26] NEWMAN M.E.J. The structure and function of networks. Comp. Phys. Communication 2002, 147, pp. 40-45, doi: 10.1016/S0010-4655(02)00201-1.

[27] PORTILLO I.J.G., GLEISER P.M. An adaptive complex network model for brain functional networks. PLoS One 2009, 4(9), e6863, doi: 10.1371/journal.pone. 0006863.

[28] RUdOlF B., MARKOŠOVÁ M., ČAJÁGI M., TIŇO, P. Degree distribution and scaling in the connecting-nearest-neighbors model. Phys. Rev. E 2012, 85, 026114, doi: 10.1103/ PhysRevE. 85.026114.

[29] SHATTUCK D.W., LEAHY R.M. BrainSuite: An automated cortical surface identification tool. Medical Image Analysis 2002, 6(2), pp. 129-142, doi: 10.1016/s1361-8415(02)00054-3. 


\section{Neural Network World 5/2020, 309-332}

[30] SCHOLZ J., DEJORI M., STETTER M., GREINER M. Noisy scale-free networks. Physica A 2005, 350(2-4), pp. 622-642, doi: 10.1016/j.physa.2004.11.012.

[31] SMITH S. M., JENKINSON M., WOOLRICH M.W., BECKMANN C.F., BEHRENS T.E.J., JOHANSEN-BERG H., BANNISTER P.R., De LUCA M., DROBNJAK I., FLITNEY D.E., NIAZY R.K., SAUNDERS J., VICKERS J., ZHANG Y., De STEFANO N., BRADY J.M., MATTHEWS P.M. Advances in functional and structural MR image analysis and implementation as FSL. Neuroimage 2004, 23 Suppl 1, pp. S208-219, doi: $1.1061 / j$.neuroimage 2004.07 .051$.

[32] SPORNS O. Networks of the Brain. Cambridge, MA: MIT Press, 2011, doi: 10.7551/ mitpress/8476.001.0001.

[33] TRIPOLITI E.E., FOTIADIS D.I., ARGYROPOLOU M., MANIS G. A six stage approach for the diagnosis of the Alzheimer's disease based on fMRI data. J. Biomed. Inform. 2010, 43(2), pp. 307-320, doi: 10.1016/j.jbi.2009.10.004.

[34] WATTS D.J., STROGATZ S.H. Collective dynamics of "small world" networks. Nature 1998, 393, pp. 440-442, doi: 10.1038/30918.

[35] WEST D.B. Introduction to Graph Theory (second edition). London: Prentice Hall, 2001. 